\title{
Diversidad genética y heredabilidad en sentido amplio en agropiro alargado, Thinopyrum ponticum
}

\author{
Susana M. Pistorale ${ }^{1}$, Liliana A. Abbott ${ }^{1}$ y Adriana Andrés ${ }^{2}$ \\ 'Departamento de Ciencias Básicas. Universidad Nacional de Luján, CC 221, (6700) Luján, \\ Buenos Aires, Argentina. \\ ${ }^{2}$ Estación Experimental Agropecuaria. INTA, Pergamino, Ruta 32, Km 4,5 (2700), Pergamino, \\ Buenos Aires, Argentina.
}

\begin{abstract}
S.M. Pistorale, L.A. Abbott, and A. Andrés. 2008. Genetic diversity and broad sense heritability in tall wheatgrass (Thinopyrum ponticum). Cien. Inv. Agr. 35(3):259-264. Ten naturalized populations of tall wheatgrass (Thinopyrum ponticum) were evaluated in a completely randomized block design with three replicates. Each plant was analyzed for morphological and reproductive characters, and statistical and genetic characters were estimated. Significant differences $(\mathrm{p}<0.001)$ were obtained for the means of all ten populations, indicating great variability between tall wheatgrass populations. The results show that the environmental coefficients of variation were high for total seed production $(69.24 \%)$, number of tillers $(54.93 \%)$, number of spikes $(48.50 \%)$, and dry matter production $(49.01 \%)$. Coefficients of variation were rather low for spike length $(19.11 \%)$ and the 1000 -seed weight $(18.44 \%)$. Heritability was high for seed weight $(0.86)$, dry matter production $(0.55)$, and the number of tillers (0.55). Intermediate heritability was found for total seed production $(0.30)$, and low heritability for the number (0.19) and length (0.09) of spikes. Even though the number of spikes showed an intermediate heritability, it was highly and positively correlated with seed production $(\mathrm{r}=0.603)$, and a little less so with seed weight $(\mathrm{r}=0.177)$. Seed weight showed a high heritability and was positively correlated with seed production $(r=0.285)$. The wide variability observed between populations for almost all the studied characters will allow them to be integrated into a single germoplasm or into a genetic pool that could be used in programs aimed at the genetic improvement of tall wheatgrass.
\end{abstract}

Key words: Genetic variability, heritability in broad sense, phenotypic correlation, tall wheatgrass.

\section{Introducción}

El agropiro alargado (Thinopyrum ponticum (Podp) Barkworth et Dewey) (sin. Agropyron elongatum (Host) Beauv, Elytrigia pontica (Podp) Holub), es una gramínea forrajera perenne introducida en Argentina. Presenta un crecimiento cespitoso, con un macollaje abundante, formando matas muy densas que mide entre 70 a $180 \mathrm{~cm}$ de altura cuando está florecida. Su sistema radical es homogéneo, poderoso y profundizante. Sus hojas son largas,

Recibido 21 diciembre 2007. Aceptado 13 junio 2008.

${ }^{1}$ Dirigir correspondencia a S.M. Pistorale: genetica@mail.unlu.edu.ar de menos de $10 \mathrm{~mm}$ de ancho, rígidas, verdes y glaucas. La inflorescencia es una espiga dística de 12 a $25 \mathrm{~cm}$ de largo con espiguillas con 5 a 10 flores. Presenta cariopsis linear y fusiforme. Produce forraje durante todo el año, pero su máxima producción ocurre en primavera. Se utiliza para heno y ensilaje (Asay y Knowles, 1985).

El agropiro alargado es uno de los pastos más tolerantes a la salinidad de los suelos (McGuire y Dvorak, 1981; Rogers y Bailey, 1963). Es una especie forrajera muy adecuada para suelos húmedos, anegados durante el invierno y secos con formación de costra superficial en verano (Oram, 1990). En Argentina, es la gramínea 
forrajera naturalizada más cultivada en suelos con limitaciones por alcalinidad y/o salinidad.

Las especies forrajeras de importancia agronómica, nativas y/o naturalizadas, están adaptadas a condiciones específicas del ambiente. Por lo tanto, constituyen un recurso disponible para áreas marginales, a menudo insuficientemente explotadas. Existen ejemplos de introducción de especies, que sin mejora genética, han demostrado un comportamiento superior al de las variedades forrajeras actualmente en uso (Burton,1979).

Uno de los atributos evolutivos más importantes de las especies nativas y naturalizadas, es la adaptación a condiciones ambientales y de manejo extremas en zonas marginales de Argentina. Por ejemplo, adaptación a suelos anegables o secos, con altas concentraciones salinas, poco fértiles, bajo condiciones de sobre pastoreo o sub pastoreo. Esta característica evolutiva, ha promovido una importante variabilidad genética y plasticidad fenotípica en la mayoría de las especies del pastizal argentino. Por lo tanto, la colección de ecotipos adaptados a diferentes condiciones ecológicas y de manejo es una fuente de germoplasma que puede proveer la variabilidad y plasticidad necesarias para desarrollar futuros programas de mejoramiento genético en especies con valor forrajero (Duyvendak y Luesink,1979).

La cuenca del Río Salado, que cruza la provincia de Buenos Aires de este a noroeste, es una de las zonas marginales para la agricultura argentina con una extensión de aproximadamente 15 millones de hectáreas, situadas dentro de la región pampeana. Es una zona templada, con promedios anuales que varían entre $16^{\circ} \mathrm{C}$ en el norte y $14^{\circ} \mathrm{C}$ en el sur. El régimen pluviométrico es irregular, con un promedio de $1000 \mathrm{~mm}$ anuales, concentradas en enero. Los suelos presentan una capa arable muy delgada, bajo contenido de materia orgánica y $\mathrm{pH}$ mayor a 8,5 en la superficie del suelo. Se clasifican en capacidad de uso Clase VI ws, asociados a suelos con problemas de drenaje, alcalinidad y sodicidad, lo que determina que sean suelos sólo aptos para ganadería.

El objetivo de este trabajo fue evaluar la variabilidad fenotípica y genotípica interpoblacional, estimar la heredabilidad en sentido amplio y las correlaciones fenotípicas en caracteres de interés agronómico en poblaciones naturalizadas de agropiro alargado, colectadas en la Cuenca del río Salado, Argentina.

\section{Materiales y métodos}

\section{Origen de la semilla}

En marzo de 2003, se recolectaron espigas maduras, en forma masal, de diez poblaciones de agropiro alargado en la Cuenca del río Salado, provincia de Buenos Aires (Figura 1). Las semillas provenientes de estas espigas se sembraron en invernáculo en mayo. Noventa plantas por cada población se transplantaron espaciadamente al alcanzar el estado de tres macollos, en el campo experimental de la Estación Experimental Agropecuaria del INTA, en Pergamino, en el centro oeste de la provincia de Buenos Aires.

\section{Parámetros evaluados}

Se evaluaron los siguientes caracteres por planta: 1. número de macollos vegetativos, 2. número de espigas, contadas a medida que se cosechaban, 3. largo de la espiga, medido desde la inserción hasta el extremo, 4. producción total de semillas llenas, en gramos, 5. peso de 1000 semillas, y 6 . producción de materia seca.

Las espigas se cosecharon a medida que maduraron y se guardaron en bolsas de papel hasta su evaluación en el laboratorio. El largo de la espiga fue el promedio de tres espigas por planta. El peso de la materia seca se obtuvo de la suma de tres cortes estacionales de materia verde (otoño, invierno y primavera), después de secados en estufa de aire forzado a $80^{\circ} \mathrm{C}$, hasta peso constante.

\section{Diseño, análisis estadísticos y genéticos}

Los caracteres se analizaron según un diseño de bloques completos al azar con diez tratamientos (poblaciones de agropiro alargado), tres repeticiones donde la unidad experimental estuvo representada por una planta. Se utilizó el programa InfoStat (Estadística y Biometría, 
Facultad de Ciencias Agropecuarias, Universidad Nacional de Córdoba, Argentina).

Se determinaron las varianzas genéticas, ambiental y genotípica mediante análisis de varianza según las ecuaciones más abajo indicadas $\mathrm{Se}$ determinó el coeficiente de variabilidad y la heredabilidad en sentido amplio.

Varianza genética $\left(\sigma_{G}^{2}\right)=C M_{p}-C_{e} / r$

Varianza ambiental $\left(\sigma_{\mathrm{A}}^{2}\right)=\mathrm{CM}_{\mathrm{e}}$

Varianza fenotípica $\left(\sigma_{\mathrm{F}}^{2}\right)=\sigma_{\mathrm{G}}^{2}+\sigma_{\mathrm{A}}^{2}$

donde: $\mathrm{CM}_{\mathrm{p}}=$ cuadrado medio de las poblaciones, $\mathrm{CM}_{\mathrm{e}}=$ cuadrado medio del error experimental, y $\mathrm{r}=$ número de repeticiones.

Los coeficientes de variación genético, fenotípico y ambiental se calcularon según:

Coeficiente de variación genético $(\mathrm{CVG})=\frac{\sqrt{ } \sigma_{\mathrm{G}}^{2} \times 100}{\bar{\chi}}$

Coeficiente de variación fenotípico $(\mathrm{CVF})=\frac{\sqrt{ } \sigma_{\mathrm{F}}^{2} \times 100}{\bar{\chi}}$

Coeficiente de variación ambiental $(\mathrm{CVA})=\frac{\sqrt{ } \sigma_{\mathrm{A}}^{2} \times 100}{\bar{\chi}}$

Con las estimaciones de los componentes de las varianzas obtenidos a partir del análisis de varianza, se estimó la heredabilidad en sentido amplio (grado de determinación genético) (Shing et al., 1993; Burton y De Vane, 1953) a partir de la siguiente expresión:

$$
\text { Heredabilidad }\left(\mathrm{h}^{2}\right)=\sigma_{\mathrm{G}}^{2} / \sigma_{\mathrm{F}}^{2}
$$

donde $\sigma_{\mathrm{F}}^{2}=\sigma_{\mathrm{G}}^{2}+\sigma_{\mathrm{A}}^{2}$

Las correlaciones fenotípicas se calcularon utilizando el coeficiente de correlación de Pearson, como medida de la magnitud de la asociación lineal entre dos variables que no dependen de las unidades de medida de las variables originales.

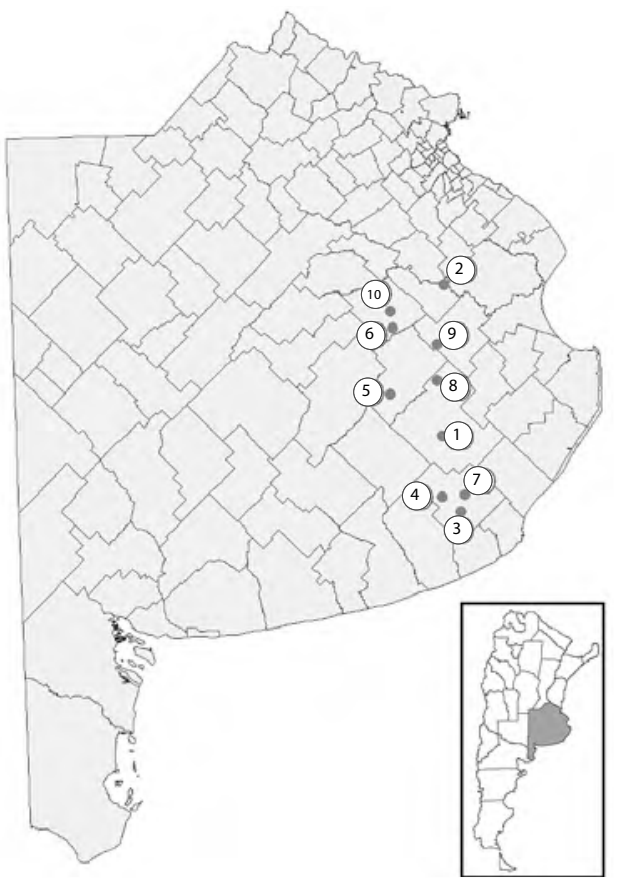

Figura 1. Sitios de recolecta de las poblaciones de agropiro alargado (Thinopyrum ponticum) en la provincia de Buenos Aires, Argentina. 1, Población Ayacucho; 2, Población General Belgrano; 3, Población Arroyo El Perdido; 4, Población Rutas 226 y 29; 5, Población Rauch; 6, Población Ruta 30 y Canal 11; 7, Población Bosh; 8 , Población Langueyú; 9, Población Casalins y 10, Población El Fortín.

Figure 1. Sites of collection of populations of tall wheatgrass (Thinopyrum ponticum) in the province of Buenos Aires, Argentina. 1. Population Ayacucho, 2. Population General Belgrano, 3. The Lost City Arroyo, 4. Town Routes 226 and 29, 5. Population Rauch, 6. Population Route 30 and Channel 11, 7. Bosh Population; 8. Population Langueyú; 9. Population Casalins and 10. The Population Fortin.

\section{Resultados y discusión}

Las comparaciones entre las medias de las diez poblaciones, que se realizaron mediante la prueba de $\mathrm{t}$, mostraron diferencias significativas ( $\mathrm{p}<0,001$ ), lo que indica la gran variabilidad existente entre las poblaciones de agropiro alargado consideradas en este estudio.

El coeficiente de variación (CV) y el coeficiente de variación ambiental (CVA) fueron altos para producción de semillas totales por planta, producción de materia seca por planta, número 
de macollos por planta y número de espigas por planta. Para peso de 1000 semillas por planta y largo de espiga por planta el CV y el CVA fueron bajos (Cuadros 1 y 2).

Los coeficientes de variación genética fueron altos para número de macollos por planta, producción de materia seca por planta, peso de 1000 semillas por planta y producción de semillas por planta. En cambio la variabilidad genética fue baja para número de espigas por planta y muy baja para largo de espiga por planta (Cuadro 2).

La relación entre el coeficiente de variación genético y ambiental (CVG / CVA) fue alto para peso de 1000 semillas por planta, número de macollos por planta y producción de materia seca por planta. Según Vencovsky (1987), existe una situación muy favorable para la ganancia por selección cuando la relación CVG / CVA tiende a uno o es superior a uno, ya que en estos casos, la variación genética es mayor que la variación ambiental, lo que indica que la selección para estos caracteres tiene las mejores condiciones en términos de ganancia genética inmediata.

De acuerdo con Stanfield (1971), los caracteres se consideraron de heredabilidad alta cuando esta fue mayor a 0,50 , de heredabilidad media entre 0,20 y 0,50 , y de heredabilidad baja si esta fue menor a 0,20. En el Cuadro 2 se observa que cuanto más alta fue la relación entre el coeficiente de variación genético y al ambiental, más alto fue el valor de la heredabilidad en sentido amplio. La heredabilidad fue alta para peso de 1000 semillas por planta, producción de materia seca por planta y número de macollos por planta. La producción total de semillas por planta presentó una heredabilidad media, pero fue baja para número de espigas por planta $\mathrm{y}$ largo de espiga por planta. La heredabilidad en sentido amplio tiene validez para fines de comparación entre caracteres e interesa para considerar las expectativas de selección existentes (Vencovsky y Barriga, 1992).

En relación a la asociación entre los caracteres evaluados, la correlación entre el número de espigas por planta y la producción total de semillas por planta fue alta y positiva $(r=$ 0,603 ). En cambio, las correlaciones fueron bajas y positivas entre producción total de semillas por planta y peso de 1000 semillas ( $\mathrm{r}$ $=0,285$ ), número de espigas y peso de 1000 semillas $(\mathrm{r}=0,177)$, número de espigas $\mathrm{y}$ producción de materia seca $(\mathrm{r}=0,177)$, largo de espiga y producción de materia seca $(\mathrm{r}=$ $0,177)$, largo de espiga y peso de 1000 semillas $(\mathrm{r}=0,146)$ y peso de 1000 y producción de materia seca por planta $(r=0,137)$. Presentaron correlaciones baja y negativa el número de macollos por planta y producción total de semillas $(r=-0,212)$, número de macollos por planta y el número de espigas por planta $(\mathrm{r}=-0,204)$ y número de macollos por planta $y$ el peso de 1000 semillas $(r=-0,162)$. Todas estas correlaciones fueron significativas $(\mathrm{p}<$ 0,001 ). Si bien el número de espigas por planta presentó una heredabilidad mediana $(0,19)$, la correlación con la producción de semillas por planta fue alta y positiva, pero mediana y positiva con peso de 1000 semillas. El peso de 1000 semillas, que presentó alta heredabilidad, correlacionó positivamente y con un valor medio, con producción de semillas por planta.

Cuadro 1. Media, valores mínimos, máximos y coeficientes de variación para diferentes caracteres en agropiro alargado (Thinopyrum ponticum).

Table 1. Mean, minimum, and maximum values and the coefficient of variation for different characters in tall wheatgrass (Thinopyrum ponticum).

\begin{tabular}{|c|c|c|c|c|}
\hline Caracteres & Media & Mínimos & Máximos & $\mathrm{CV}^{1}, \%$ \\
\hline Macollos por planta, no. & 99,75 & 12,00 & 524,00 & 56,93 \\
\hline Espigas por planta, no. & 49,37 & 1,00 & 132,00 & 48,70 \\
\hline Largo de espiga, $\mathrm{cm}$ & 29,15 & 10,00 & 52,96 & 19,21 \\
\hline Producción de semillas por planta, $g$ & 16,08 & 0,17 & 70,40 & 70,75 \\
\hline Peso de 1000 semillas, $\mathrm{g}$ & 6,30 & 2,71 & 9,60 & 20,39 \\
\hline Producción de materia seca, $\mathrm{g}$ & 145,30 & 2,40 & 888,83 & 60,39 \\
\hline
\end{tabular}

${ }^{1} \mathrm{CV}=$ coeficientes de variación calculado como el desvío estándar expresado como porcentaje de la media aritmética. 
Cuadro 2. Variancia genética $\left(\sigma_{\mathrm{G}}^{2}\right)$, ambiental $\left(\sigma_{\mathrm{A}}^{2}\right)$ y fenotípica $\left(\sigma_{\mathrm{F}}^{2}\right)$, heredabilidad en sentido amplio $\left(\mathrm{h}^{2}\right)$ y coeficientes de variación genético (CVG), fenotípico (CVF) y ambiental (CVA) para diferentes caracteres en agropiro alargado (Thinopyrum ponticum).

Table 2. Genetic $\left(\sigma_{G}^{2}\right)$, environmental $\left(\sigma_{A}^{2}\right)$ and phenotypic $\left(\sigma_{F}^{2}\right)$ variance, heritability $\left(h^{2}\right)$ in broad sense, and the genetic $(C V G)$, phenotypic $(C V F)$ and environmental $(C V A)$ coefficients of variation for different characters in tall wheatgrass (Thinopyrum ponticum).

\begin{tabular}{lccccrr}
\hline Parámetros & $\begin{array}{c}\text { Número de } \\
\text { macollos }\end{array}$ & $\begin{array}{c}\text { Número de } \\
\text { espigas }\end{array}$ & $\begin{array}{c}\text { Largo de } \\
\text { espiga }\end{array}$ & $\begin{array}{c}\text { Producción de } \\
\text { semilla }\end{array}$ & $\begin{array}{c}\text { Peso de } \\
\text { 1000 semillas }\end{array}$ & $\begin{array}{c}\text { Producción de } \\
\text { materia seca }\end{array}$ \\
\hline$\sigma^{2}{ }_{\mathrm{G}}$ & 3673,42 & 131,91 & 3,43 & 52,29 & 8,08 & 6228,76 \\
$\sigma^{2}$ & 3002,42 & 573,22 & 31,03 & 123,95 & 1,35 & 5070,27 \\
$\sigma^{2}{ }_{\mathrm{F}}$ & 6675,84 & 705,12 & 34,46 & 176,24 & 9,43 & 11299,03 \\
$\mathrm{~h}^{2}$ & 0,55 & 0,19 & 0,10 & 0,30 & 0,86 & 0,55 \\
$\mathrm{CVG}, \%$ & 60,76 & 23,26 & 6,35 & 44,97 & 45,12 & 54,32 \\
$\mathrm{CVF}, \%$ & 81,91 & 53,79 & 20,14 & 82,56 & 48,74 & 73,16 \\
$\mathrm{CVA}, \%$ & 54,93 & 48,50 & 19,11 & 69,24 & 18,44 & 49,11 \\
$\mathrm{CVG} / \mathrm{CVA}$ & 1,11 & 0,48 & 0,33 & 0,65 & 2,42 & 1,11 \\
\hline
\end{tabular}

Conocer las correlaciones entre los caracteres y la heredabilidad de los mismos, permite aumentar la eficiencia de los programas de selección.

El presente trabajo permitió detectar una importante variabilidad en caracteres de interés agronómicos en las diez poblaciones de agropiro alargado analizadas. Si bien existen numerosos trabajos realizados en Argentina sobre esta especie, estos se refieren principalmente a aspectos agronómicos como producción de forraje, respuesta a la fertilización nitrogenada y fosfatada, calidad del forraje, producción de semilla y manejo del pastoreo, pero son escasos los estudios sobre la variación genética presente en esta especie.
La amplia variabilidad detectada entre las poblaciones de agropiro alargado para la casi totalidad de los caracteres estudiados, permitirá integrarlas en un sólo germoplasma base para ser utilizado en programas de mejoramiento genético de agropiro alargado.

\section{Resumen}

Diez poblaciones naturalizadas de agropiro alargado (Thinopyrum ponticum) se evaluaron en un ensayo en bloques completamente aleatorizados, con tres repeticiones. Se analizaron, caracteres morfológicos y reproductivos por planta individual y se estimaron parámetros estadísticos y genéticos. Las comparaciones entre medias de las diez

Cuadro 3. Correlaciones fenotípicas entre caracteres productivos en agropiro alargado (Thinopyrum ponticum). Table 3. Phenotypic correlation between the characters in tall wheatgrass (Thinopyrum ponticum).

\begin{tabular}{|c|c|c|c|c|c|}
\hline Caracteres & $\begin{array}{l}\text { Macollos } \\
\text { por planta }\end{array}$ & $\begin{array}{l}\text { Espigas } \\
\text { por planta }\end{array}$ & $\begin{array}{l}\text { Largo } \\
\text { de espiga }\end{array}$ & $\begin{array}{c}\text { Producción de } \\
\text { semilla por planta }\end{array}$ & $\begin{array}{c}\text { Peso } 1000 \\
\text { semillas }\end{array}$ \\
\hline Espigas por planta & $\begin{array}{r}-0,204^{1} \\
(<0,000)\end{array}$ & & & & \\
\hline Largo de espiga & $\begin{array}{c}0,054 \\
(0,121)\end{array}$ & $\begin{array}{r}0,062^{1} \\
(0,076)\end{array}$ & & & \\
\hline Producción de semilla por planta & $\begin{array}{c}-0,212 \\
(<0,000)\end{array}$ & $\begin{array}{c}0,603 \\
(<0,000)\end{array}$ & $\begin{array}{r}0,097^{1} \\
(0,006)\end{array}$ & & \\
\hline Peso 1000 semillas & $\begin{array}{c}-0,162 \\
(<0,000)\end{array}$ & $\begin{array}{c}0,177 \\
(<0,000)\end{array}$ & $\begin{array}{c}0,146 \\
(<0,000)\end{array}$ & $\begin{array}{r}0,285^{1} \\
(<0,000)\end{array}$ & \\
\hline $\begin{array}{l}\text { Producción de materia seca } \\
\text { por planta }\end{array}$ & $\begin{array}{l}-0,034 \\
(0,329)\end{array}$ & $\begin{array}{c}0,177 \\
(<0,000)\end{array}$ & $\begin{array}{c}0,177 \\
(<0,000)\end{array}$ & $\begin{array}{l}-0,011 \\
(0,755)\end{array}$ & $\begin{array}{r}0,137^{1} \\
(<0,000)\end{array}$ \\
\hline
\end{tabular}

${ }^{1}$ Coeficientes de correlación según Pearson, en paréntesis se indica la probabilidad.

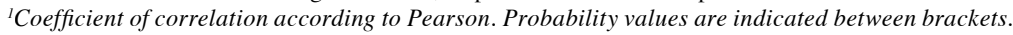


poblaciones, mediante la prueba de $t$, mostraron diferencias significativas ( $p<0,001$ ), lo que indica la gran variabilidad existente entre las poblaciones. Los resultados mostraron que los coeficientes de variación ambiental fueron altos para producción de total de semilla $(69,24 \%)$, número de macollos (54,93\%), número de espigas $(48,50 \%)$ y producción de materia seca $(49,01 \%)$, y fueron bajos para largo de espiga $(19,11 \%)$ y peso de 1000 semillas $(18,44 \%)$. La heredabilidad fue alta para peso de 1000 semillas $(0,86)$, producción de materia seca $(0,55)$ y número de macollos $(0,55)$. Presentó heredabilidad media la producción total de semillas $(0,30)$, y heredabilidad baja el número deespigas $(0,19)$ y el largo de espiga $(0,10)$. Sibien el número de espigas presentó una heredabilidad mediana, la correlación con la producción de semillas fue alta y positiva, y con peso de 1000 semillas fue mediana y positiva. El peso de 1000 semillas, que presentó alta heredabilidad, correlacionó positivamente con la producción de semillas. La amplia variabilidad detectada entre las poblaciones, para la casi totalidad de los caracteres estudiados, permitirá utilizarlas en programas de mejoramiento genético de agropiro alargado.

Palabras clave: Agropiro alargado, correlaciones fenotípicas, heredabilidad en sentido amplio, variabilidad genética.

\section{Agradecimientos}

Se agradece el financiamiento recibido del Departamento de Ciencias Básicas de la Universidad Nacional de Luján, Buenos Aires, Argentina y la colaboración del personal de campo del Instituto Nacional de Tecnología Agropecuaria (INTA) de Pergamino, Buenos Aires, Argentina, para la realización de este trabajo.

\section{Literatura citada}

Asay, K.H. y R.P. Knowles. 1985. The wheatgrasses. Forages: the science of grassland agriculture. Pages 166-176. In: R.F. Barnes, D.S. Metcalfe and M.E. Heath (eds.). Iowa State University Press. Iowa, USA.

Burton, G.W. 1979. Handling cross-pollinated germplasm efficiently. Crop Science 19:695690.

Burton, G.W. y R.W. De Vane. 1953. Estimating heritability in tall Fescue (Festuca arundinacea) from replicated clonal material. Agronomy Journal 45:478-481.

Duyvendak, R. y B. Luesink. 1979. Preservation of genetic resources in grasses. Pages 67-73. In: Proceeding Conference Broadening Genetics. Base Crops, 1978. Pudoc, Wageningen, The Netherlands.

McGuire, P.E. y J. Dvorak. 1981. High salt tolerance potential in wheatgrasses. Crop Science 21:702705.

Oram, R.N. 1990. Register of Australian herbage plant cultivars, 3rd ed. CSIRO, Melbourne, Australia.

Rogers, A.L. y E.T. Bailey. 1963. Salt tolerance trials with forage plants in south Western Australia. Australian Journal of Experimental Agriculture and Animal Husbandry 3:125-130.

Shing, M., S. Ceccarelli y J. Hambling. 1993. Estimation of heretability from varietal trials data. Theorical and Applied Genetics 86:437441.

Stanfield, W.D. 1971. Genética. Teoría y 440 problemas resueltos. Segunda ed. Serie Schaum, McGraw-Hill, México. 405 pp.

Vencovsky, R. 1987. Genética Cuantitativa. In: E. Paterniani (ed.). Melhoramento e Produção do Milho no Brasil. Edição da Fundação Cargill. Instituto de Genetica, Escuela Superior de Agricultura Luis de Queiroz, Universidade de São Paulo. Piracicaba, São Paulo, Brazil.

Vencovsky, R. y P. Barriga. 1992. Genética Biométrica no fitomelhoramento, Ribeirao Preto, Revista Brasileira de Genética, 496 pp. 